\title{
Language improvement one week after thrombolysis in acute stroke
}

\author{
Martins IP, Fonseca J, Morgado J, Leal G, Farrajota L, Fonseca AC, \\ Melo TP. Language improvement one week after thrombolysis in \\ acute stroke. \\ Acta Neurol Scand 2017: 135: 339-345. \\ (C) 2016 John Wiley \& Sons A/S. Published by John Wiley \& Sons Ltd.
}

Objectives - Language recovery following acute stroke is difficult to predict due to several evaluation factors and time constraints. We aimed to investigate the predictors of aphasia recovery and to identify the National Institute of Health and Stroke Scale (NIHSS) items that best reflect linguistic performance, 1 week after thrombolysis.

Materials and methods - We retrieved data from a prospective registry of patients with aphasia secondary to left middle cerebral artery

(MCA) stroke treated with intravenous thrombolysis. Complete recovery at day 7 (D7) was measured in a composite verbal score (CVS) ( $\Sigma$ Language+Questions + Commands NIHSS scores). Lesion size was categorized by the Alberta Stroke Program Early CT score (ASPECTS) and vascular patency by ultrasound. CVS was correlated with standardized aphasia testing if both were performed within a two-day interval. Results - Of 228 patients included (age average 67.32 years, $131 \mathrm{men}$ ), $72 \%$ presented some language improvement that was complete in $31 \%$. Total recovery was predicted by ASPECTS $(\mathrm{OR}=1.65 ; 95 \% \mathrm{CI}, 1.295-2.108 ; P<0.00)$ and baseline aphasia severity $(\mathrm{OR}=0.439 ; 95 \% \mathrm{CI}, 0.242-0.796 ; P<0.007)$. CVS correlated better with standardized aphasia measures (aphasia quotient, severity, comprehension) than NIHSS_Language item. Conclusions - Lesion size and initial aphasia severity are the main predictors of aphasia recovery one week after thrombolysis. A NIHSS composite verbal score seems to capture the global linguistic performance better than the language item alone.

\author{
I. P. Martins ${ }^{1}$, J. Fonseca ${ }^{1}$, \\ J. Morgado ${ }^{2}$, G. Leal ${ }^{1,3}$, \\ L. Farrajota, ${ }^{1,3}$ A. C. Fonseca ${ }^{3,4}$, \\ T. P. Melo ${ }^{3,4}$ \\ ${ }^{1}$ Language Research Laboratory, Faculty of Medicine \\ and IMM, University of Lisbon, Lisbon, Portugal; \\ ${ }^{2}$ Department of Neurology, Centro Hospitalar Lisboa \\ Central, Lisbon, Portugal; ${ }^{3}$ Department of Neurology, \\ Hospital de Santa Maria - Centro Hospitalar Lisboa \\ Norte, Lisbon, Portugal; ${ }^{4}$ Stroke Unit, Hospital de Santa \\ Maria - Centro Hospitalar Lisboa Norte, Lisbon, \\ Portugal
}

Key words: aphasia; strokes; speech and language disorders

I. P. Martins, Language Research Laboratory, Faculty of Medicine, University of Lisbon, Av. Prof. Egas Moniz, 1649-028, Lisbon, Portugal Tel.:/Fax: 00351217934480 e-mail: ipavaomartins@gmail.com

Accepted for publication March 29, 2016

\section{Introduction}

Aphasia is one of the most disabling neurological conditions, with a significant impact on quality of life, mood, and ability to return to work (1-3). It occurs in $15-40 \%$ of individuals with left hemisphere stroke and persists in $12.5-50 \%$ (3-6). Most data on aphasia recovery were obtained before the modern era of thrombolytic therapy. However, the treatment with recombinant tissue plasminogen activator (rTPA), particularly if early recanalization is achieved, can modify the size, pattern, and nature of infarcts following ischemic stroke (7-9). As a consequence, the linguistic impairments following a stroke as well as their expected prognosis could also be different. Given the current recommendation to use intravenous thrombolysis as the standard of care in acute stroke patients fulfilling the inclusion criteria, it is important to reevaluate the factors associated with aphasia recovery.

The outcome of aphasia is difficult to predict within the first days of stroke. Several reasons and time constraints preclude the use of standardized batteries of tests for language assessment before rTPA $(5,10)$. The NIHSS (National Institute of Health and Stroke Scale) is the gold standard assessment tool to evaluate neurological deficits in acute stroke patients. Nonetheless, the language item of the scale has been criticized due to its inability to differentiate between aphasic symptoms, its poor evaluation of comprehension, and its failure to identify mild symptoms $(11,12)$. A factor analysis of the NIHSS (13) identified 


\section{Martins et al.}

two items that are loaded in the same factor as language: the request to answer some questions (item $1 \mathrm{~b}$ ) and the response to verbal commands (1c). Although not tackling language specifically, these items can be considered indirect measures of aphasia. In two recent studies $(12,14)$, they were used in a compound measure to evaluate language improvement, but so far they have not been validated as such.

In this study, we aimed to assess: (i) the validity of the NIHSS composite verbal score (CVS), comparing it to quantitative measures obtained in a standardized assessment; (ii) language recovery in the first week after thrombolysis and to identify prognostic factors among clinical and imaging variables.

\section{Material and methods}

Study design

Retrospective analysis based on a prospectively collected register of consecutive patients treated with intravenous thrombolysis for acute ischemic stroke. Data were collected in a Stroke Unit of a University Hospital according to a standard protocol between 2003 and 2014 and concerns two time points - on admission and on day seven (D7). The study protocol was approved by the joint Ethics Committee of the Faculty and Hospital.

\section{Population}

Records of consecutive patients with aphasia due to ischemic stroke of the left middle cerebral artery (MCA) territory admitted to a Stroke Unit for thrombolysis were selected. Aphasia was diagnosed by the attending neurologist, with experience in stroke based on a bedside language evaluation usually comprising spontaneous speech, naming and comprehension of commands. Any score $\geq 1$ on the NIHSS Language item (item 9) was diagnostic of aphasia. All patients had two language evaluations, on admission (before thrombolysis) and on D7. Attending neurologists and residents have identical expertise in the NIHSS scale and participate in clinical trials that demand certification in the NIHSS scale. We excluded patients who did not complete the second evaluation due to death, unconsciousness, or transfer to other units, with the exception of those who were discharged before D7 without any language impairment, who were assigned a D7 language score of zero. Patients did not initiate Speech and Language Therapy before D7.
Aphasia severity was measured in a composite verbal score (CVS), ranging 0-7, corresponding to the sum of three NIHSS items: item 9 (NIHSS_best Language) with four possible degrees $(0=$ no aphasia or normal; $1=$ mild to moderate aphasia; $2=$ severe aphasia, and $3=$ mute/global aphasia); item 1b (NIHSS_Questions), scoring 0,1 , or 2 (answers two questions correctly, one or none, respectively); and item 1c (NIHSS_Commands), scoring 0, 1, or 2 (performing both, one, or none correctly). Complete aphasia recovery was defined as a D7 CVS of zero. Aphasia improvement was defined as any decrease of one or more points in CVS between admission and D7.

\section{Procedures}

Demographic (age, gender, literacy) and clinical data were retrieved from the registry, including vascular risk factors, onset to needle time, baseline and D7 neurological status (total NIHSS score and verbal and right motor subscores), left internal carotid artery (ICA), and MCA patency verified by carotid and transcranial ultrasound, hemorrhagic transformation of the infarct, and modified Rankin scale at discharge.

All patients undertook an unenhanced brain CT scan before thrombolysis and received rTPA within the first 3 (between 2003 and 2010) or $4.5 \mathrm{~h}$ (2010-2014) of stroke onset. Subsequent brain imaging (CT or MRI) was performed in cases with no visible lesion in the acute CT, with suspected hemorrhagic transformation or other clinical reasons. Carotid Ultrasound and Transcranial Doppler were used to assess patency of the left MCA and ICA between 12 and $72 \mathrm{~h}$.

Brain imaging analysis

Neuroimaging data were retrieved from medical records. Lesions were analyzed on the first CT or MRI performed after thrombolysis or on admission CT if there were no subsequent images. The analysis was performed by two independent examiners, blind to the clinical information, according to the Alberta Stroke Program Early CT Score (ASPECTS) (15) for the presence or absence of lesion in ten different areas of the left MCA territory. Affected regions are summed, and the total is subtracted from 10 , producing a score ranging between 0 (lesion in all MCA territory) and 10 (no visible lesion). Every time there was no complete agreement, the images were reviewed and scored by consensus. 


\section{Standardized aphasia evaluation}

Patients with persisting aphasia were also evaluated during admission by Speech and Language therapists using a standardized aphasia battery, the Lisbon Aphasia Assessment Battery (BAAL) (16), with tests of speech fluency, object naming, word and sentence repetition (producing a repetition score), object identification and comprehension of simple commands (producing a comprehension score) and two independent measures of aphasia severity: the Aphasia Severity Rating Scale (ASRS) of the Boston Diagnosis Aphasia Examination (17) and the aphasia quotient $(\mathrm{AQ}=$ the mean of correct percentage obtained in four BAAL subtests (fluency, naming, word repetition and comprehension of commands)). AQ is comparable its counterpart of the Western Aphasia Battery $(18,19)$.

Composite verbal score and the composing NIHSS scores at D7 were correlated with standard aphasia measures to evaluate their reliability. Assuming that aphasia severity suffers significant changes in the first days after stroke, correlations were calculated only for patients who undertook aphasia assessment between the day five and day nine $(\mathrm{D} 7 \pm 2)$.

\section{Statistical analysis}

Demographic, clinical, and imaging data were analyzed by descriptive statistics. Interrater reliability was evaluated by $\mathrm{k}$ statistics. Biserial point correlations were calculated between NIHSS scores and language tests scores. Patients were classified as recovered or not recovered (D7_CVS of 0 or $\geq 1$, respectively), and the clinical and imaging features were compared between groups, by Student's $t$-test or Mann-Whitney $U$ test for continuous variables and chi-square for nonparametric variables. Factors considered relevant for recovery $(P \leq 0.15$ in univariate analysis) were entered in a multivariable logistic regression. Odd ratios and 95\% CI were calculated.

The degree of CVS improvement was explored by linear regression analysis. As initial aphasia severity modulates the amount of possible recovery (more limited in low initial scores), improvement was calculated separately for mild aphasia (NIHSS_Language $=1$ ) and severe/global aphasia (NIHSS_Language $>1$ ) at baseline. Statistical analyses were performed using SPSS software package (SPSS version 21.0 for Windows, NY, USA). All statistical tests were two sided with significant levels at alpha $<0.05$.

\section{Results}

A total of 624 patients were admitted with aphasia due to acute MCA ischemic stroke, and 252 had undergone thrombolysis. Sixteen patients died, six were transferred to other units without a second evaluation, two were excluded because the lesion was on the right hemisphere, and 228 cases were included.

Patients were on average 68.3 years of age (range 25-87) and 57.5\% were male patients. Mean CVS at entry was 5.15 with a median of 6 (range 1-7) (Table 1). Brain imaging was analyzed in 182 cases $(79.8 \%)$, and 33 of these on MRI. In 46 earlier cases, imaging exams were not permanently stored. Cases without brain imaging did not differ from the others in demographics, risk factors, onset to needle time, baseline NIHSS (Total, best Language and CVS score), vessel patency, aphasia recovery, or improvement. Interrater agreement of ASPECTS score was 0.953 (Cohen Kappa, $P<0.000$ ). The median value of ASPECTS was 7 (ASPECTS $<7$ in $86(47.5 \%$ ) patients). There was a negative but nonsignificant correlation between initial CVS and ASPECT score (Spearman's rho $=-0.064, \mathrm{~ns}$ ). The majority of patients had a single lesion of the left hemisphere but 24 presented previous vascular lesions, 16 on the right hemisphere. Hemorrhagic transformation occurred in $22.2 \%$.

\section{Aphasia recovery}

By D7, 30.7\% $(N=70)$ of patients had recovered completely and $71.9 \%(N=164)$ presented some degree of improvement in the CVS. The majority (117) improved between 1 and 4 points, but 47 patients improved more than four points, including seven cases that improved seven points. Fifty cases remained unchanged and 14 worsened.

Differences between recovery groups are shown in Table 1. Patients who recovered had lower NIHSS_total, milder aphasia (CVS and NIHSS best Language) and less right limb weakness (NIHSS score sum for right motor arm and leg) at baseline. In addition, they also had smaller infarcts and more often presented vessel patency and absence of hemorrhagic transformation.

A multivariate logistic regression analysis (Table 2) revealed that ASPECTS and baseline aphasia severity explained $45.7 \%$ of total recovery. The degree of CVS improvement (linear regression analysis) was related to ASPECTS, age, and initial aphasia severity, but the model was only significant for patients with a baseline NIHSS best Language $>1$. Figure 1 shows the 


\section{Martins et al.}

Table 1 Comparisons between patients with or without total aphasia recovery

\begin{tabular}{|c|c|c|c|c|}
\hline & \multirow[b]{2}{*}{ Total } & \multicolumn{2}{|c|}{ Complete recovery } & \multirow[b]{2}{*}{$P$-value } \\
\hline & & No & Yes & \\
\hline N & 228 & 158 & 70 & \\
\hline Age (sd) & $68.32 \pm 11.31$ & $68.44 \pm 11.89$ & $68.03 \pm 9.94$ & 0.79 \\
\hline Literacy (sd) & $6.04 \pm 5.24$ & $6.22 \pm 5.27$ & $2.00 \pm 2.00$ & 0.17 \\
\hline Gender (M:F) & $131: 97$ & $91: 67$ & $40: 30$ & 0.95 \\
\hline \multicolumn{5}{|l|}{ Hypertension } \\
\hline No & 86 & 56 & 30 & \multirow[t]{2}{*}{0.29} \\
\hline Yes & 142 & 102 & 40 & \\
\hline \multicolumn{5}{|l|}{ Diabetes } \\
\hline No & 169 & 116 & 53 & \multirow[t]{2}{*}{0.72} \\
\hline Yes & 59 & 42 & 17 & \\
\hline \multicolumn{5}{|l|}{ Dyslipidemia } \\
\hline No & 153 & 104 & 49 & \multirow[t]{2}{*}{0.54} \\
\hline Yes & 75 & 54 & 21 & \\
\hline BL NIHSS_Total & $15.23 \pm 6.14$ & $16.98 \pm 5.61$ & $11.27 \pm 5.41$ & $<0.001$ \\
\hline \multicolumn{5}{|c|}{ BL NIHSS_Language } \\
\hline 1 & 38 & 13 & 25 & \multirow[t]{3}{*}{$<0.001$} \\
\hline 2 & 105 & 71 & 34 & \\
\hline 3 & 85 & 74 & 11 & \\
\hline BL-CVS & $5.15 \pm 1.77$ & $5.56 \pm 1.62$ & $4.24 \pm 1.78$ & $<0.001$ \\
\hline \multicolumn{5}{|c|}{ BL Right Limb Weakness } \\
\hline No & 32 & 15 & 17 & \multirow[t]{2}{*}{0.003} \\
\hline Yes & 196 & 143 & 52 & \\
\hline Onset needle & $150.6 \pm 41.66$ & $149.81 \pm 45.67$ & $152.37 \pm 31.02$ & 0.62 \\
\hline \multicolumn{5}{|l|}{ Vessel patency } \\
\hline No & 103 & 83 & 20 & \multirow[t]{2}{*}{0.001} \\
\hline Yes & 124 & 75 & 49 & \\
\hline ASPECTS Total & $6.25 \pm 2.70$ & $5.37 \pm 2.67$ & $8.23 \pm 1.41$ & $<0.001$ \\
\hline \multicolumn{5}{|l|}{ Single infarct } \\
\hline No & 24 & 18 & 6 & \multirow[t]{2}{*}{0.43} \\
\hline Yes & 158 & 108 & 50 & \\
\hline \multicolumn{5}{|c|}{ Previous Right hemisphere lesion } \\
\hline No & 166 & 115 & 51 & \multirow[t]{2}{*}{0.002} \\
\hline Yes & 16 & 11 & 5 & \\
\hline \multicolumn{5}{|c|}{ Hemorrhagic transformation } \\
\hline No & 144 & 92 & 52 & \multirow[t]{2}{*}{$<0.001$} \\
\hline Yes & 41 & 37 & 4 & \\
\hline
\end{tabular}

Sd, standard deviation; M, male; F, female; BL, baseline; NIHSS, National Institute of Health and Stroke Scale; CVS, composite verbal score; ASPECTS, Alberta Stroke Program Early CT score.

pattern of improvement between baseline and D7 for each degree of initial aphasia and lesion size categorized as small (ASPECTS $\geq 7$ ) or large (ASPECTS <7).

\section{Reliability of NIHSS verbal scores}

Thirty-four patients undertook a standardized aphasia evaluation between the day five and day nine coinciding with NIHSS 7D assessment \pm 2 days. Correlations between the NIHSS scores and aphasia measures (Table 3) were significant and highest between the CVS and global measures of aphasia severity (AQ, BASR) or verbal comprehension. NIHSS_Commands had a higher correlation with comprehension measures than best Language or Questions items. And each NIHSS_Commands category corresponds to a different comprehension score in the standard Language Assessment, that ranges from 0 to 24 (score $0=20.2 ;$ score $1=14.4 ;$ score $2=7.3$; ANOVA $\mathrm{F}=8.62 ; P=0.001$ ).

The three items, composing CVS, presented an internal consistency of 0.723 . There was a considerable overlap in the definition of total recovery in NIHSS_best Language and CVS on D7, yet two patients fully recovered according to NIHSS_best Language were considered impaired in CVS.

\section{Discussion}

In this study, we analyzed the outcome of acute aphasia seven days after thrombolysis for acute stroke. One-third of the patients recovered completely and another $40 \%$ presented some degree of language improvement. Lesion size (ASPECTS 
Table 2 Multivariate regression analysis

\begin{tabular}{|c|c|c|c|c|c|}
\hline & \multirow[b]{2}{*}{ Adjusted $\mathrm{R}^{2}$} & \multirow[b]{2}{*}{$P$} & \multirow[b]{2}{*}{ OR } & \multicolumn{2}{|c|}{$95 \% \mathrm{Cl}$} \\
\hline & & & & Lower & Upper \\
\hline \multicolumn{6}{|l|}{$\begin{array}{l}\text { Complete Aphasia } \\
\text { recovery }\end{array}$} \\
\hline ASPECTS Score & 0.457 & 0.000 & 1.652 & 1.295 & 2.108 \\
\hline $\begin{array}{l}\text { Baseline } \\
\text { NIHSS_Language }\end{array}$ & & 0.007 & 0.439 & 0.242 & 0.796 \\
\hline Vessel patency & & 0.049 & 0.432 & 0.187 & 0.998 \\
\hline $\begin{array}{l}\text { Hemorrhagic } \\
\text { transformation }\end{array}$ & & $0.156, \mathrm{~ns}$ & 2.449 & 0.710 & 8.444 \\
\hline $\begin{array}{l}\text { Baseline } \\
\text { NIHSS_R Limb } \\
\text { weakness }\end{array}$ & & $0.601, \mathrm{~ns}$ & 1.305 & 0.482 & 3.531 \\
\hline \multicolumn{6}{|c|}{ Degree of CVS improvement (cases with baseline aphasia $>1$ ) } \\
\hline ASPECTS Score & 0.407 & 0.000 & 0.603 & 0.387 & 0.628 \\
\hline Vessel patency & & $0.074, \mathrm{~ns}$ & 0.122 & -0.055 & 1.160 \\
\hline Age & & 0.009 & -0.170 & -0.063 & -0.009 \\
\hline $\begin{array}{l}\text { Baseline } \\
\text { NIHSS_Language }\end{array}$ & & 0.006 & 0.184 & 0.244 & 1.448 \\
\hline $\begin{array}{l}\text { Baseline } \\
\text { NIHSS_R limbs }\end{array}$ & & $0.345, \mathrm{~ns}$ & -0.065 & -1.355 & 0.477 \\
\hline
\end{tabular}

NIHSS, National Institute of Health and Stroke Scale; CVS, composite verbal score; ASPECTS, Alberta Stroke Program Early CT score; R, right; D7, 7th day post onset.

score) and baseline aphasia severity were the main predictors of total recovery. The degree of aphasia recovery between admission and D7 was inversely related to lesion size, age, and initial aphasia severity.

Each of the NIHSS items related to language (best Language, Commands, and Questions) presented significant correlation with standardized aphasia measures on D7, but their sum in a Composite Verbal Score correlated better with those measures. Those three items presented a good internal consistency between them, supporting previous studies showing that they are measuring identical functions (13). To note that NIHSS_Commands correlated better with language comprehension than NIHSS_best Language, thus adding another linguistic dimension to language assessment, not completely reflected in NIHSS_best Language. Despite being a rough measure with an insufficient number of items, NIHSS_Commands gives explicit information about auditory verbal comprehension. These results, together with the factor analytic structure of NIHSS (13), support the use of this composite score as an indicator of aphasia severity although its validity as a diagnostic tool needs to be determined. This score can be particularly relevant for aphasia assessment given the widespread use of the NIHSS scale, allowing direct comparisons between different series of stroke patients.
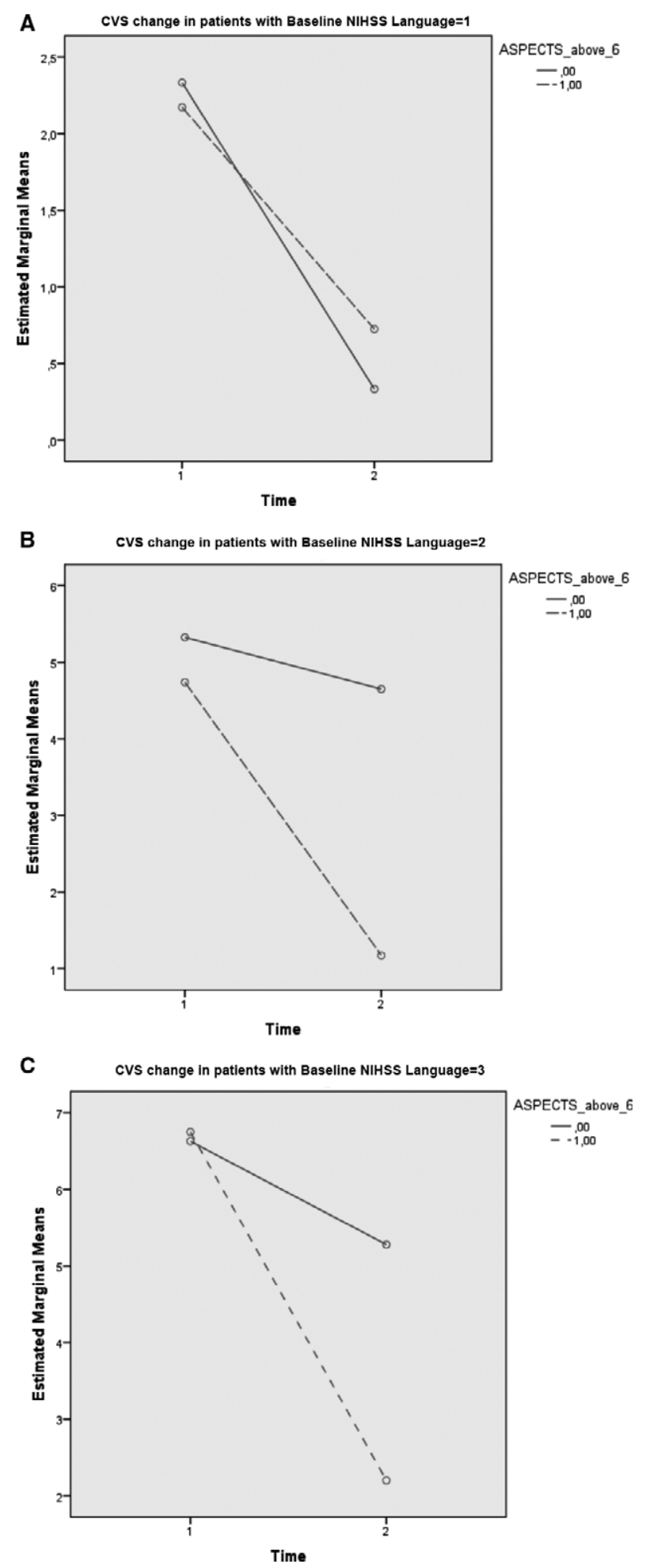

Figure 1. Composite verbal score improvement between admission (Time 1) and D7 (Time 2). Different lines correspond to lesion size in ASPECTS (bold line ASPECTS $\geq 7$; dotted line ASPECTS $<7$ ). Figures correspond to initial aphasia severity (NIHSS_Language score): A-mild aphasia; B-moderate/severe aphasia; C-global aphasia/mutism. 
Table 3 Correlations between NIHSS language and composite verbal scores and standardized aphasia evaluation $(N=34)$

\begin{tabular}{lllll}
\hline & CVS & $\begin{array}{c}\text { NIHSS } \\
\text { Language }\end{array}$ & $\begin{array}{c}\text { NIHSS } \\
\text { Commands }\end{array}$ & $\begin{array}{c}\text { NIHSS } \\
\text { Questions }\end{array}$ \\
\hline A0 & $-0.544^{* *}$ & $-0.388^{*}$ & $-0.496^{* *}$ & $-0.428^{*}$ \\
BASRS & $-0.660^{* *}$ & $-0.537^{* *}$ & $-0.471^{* *}$ & $-0.594^{* *}$ \\
Fluency & $-0.453^{* *}$ & $-0.415^{*}$ & -0.144 (ns) & $-0.539^{*}$ \\
Naming & $-0.409^{*}$ & -0.268 (ns) & $-0.431^{*}$ & -0.258 (ns) \\
Comprehension Score & $-0.583^{* *}$ & $-0.450^{* *}$ & $-0.609^{* *}$ & -0.342 \\
Repetition Score & $-0.369^{*}$ & -0.242 (ns) & -0.217 (ns) & $-0.400^{*}$ \\
\hline
\end{tabular}

A0, aphasia quotient; BASRS, Boston aphasia severity rating; CVS, composite verbal score; NIHSS, National Institute of Health and Stroke Scale.

${ }^{* *} P$-value $<0.01$

${ }^{*} P$-value $<0.05$

Two previous studies with CVS $(12,14)$ including 129 left MCA cases and 100 cases with isolated aphasia, produced similar degrees of improvement, but did not take lesion size into account.

Language is a complex function, and improvement following stroke depends on different recovery and compensatory mechanisms deployed at different times. Functional reorganization is likely the main mechanism supporting language improvement in the subacute and chronic stages of aphasia $(20,21)$ - a process that can be enhanced by speech and language therapy as well as by other interventions acting on brain plasticity $(22,23)$. However, the mechanisms involved in acute recovery seem to be more related to the hemodynamic changes (24-26) that will determine the extension and nature of the ischemia or infarct, and possibly its distant effects on normal tissue. Thrombolysis contributes to this early recovery. Indeed, a retrospective observational study of two patient groups (those who received vs. those who did not receive thrombolysis) has shown that rtPA treatment improves the prognosis of aphasia at one week and three months after the stroke (27). Moreover, recanalization is associated with a decrease of several NIHSS scores at $24 \mathrm{~h}$, including those composing the CVS (25). The contribution of recanalization/vessel patency to language improvement, in our study, also supports the role of hemodynamic mechanisms on the acute outcome. However, it is worth noticing that 49 cases had no vessel patency in the group of patients with complete recovery. The improvement observed in those cases may then be related to the collateral circulation, which restores perfusion with a reversion of initial diaschisis and ischemic penumbra.

Lesion size is particularly important in the acute and subacute stages (11, 13), suggesting that immediate language recovery depends more on intact areas of the left hemisphere than those of the right one. Indeed, the presence of previous areas of infarct in the right hemisphere did not modify the immediate outcome of aphasia in the present study. Lesion measurement is therefore important, and our semi-quantitative assessment of lesion size proved to be not only reliable, but also useful for the prognosis. However, it did not account for all variance in recovery or improvement. Among the variables not measured was diaschisis, a reversible metabolic depression observed in structurally unaffected areas of both cerebral hemispheres in the acute stage of stroke (28). Its reversibility may explain some degree of functional recovery. The poor correlation found on admission between aphasia severity (measured by the CVS) and the ASPECT score suggests that lesion alone does not account for aphasia severity and supports that diaschisis could play a role in the initial language impairment. On the other hand, patients with mild aphasia tend to have a good outcome independent of lesion size, which is usually small. In the present series, lesion size, among patients with mild aphasia (NIHSS_best Language $=1$ at entry), was five in ASPECTS, and most cases $(60 \%)$ scored between 7 and 9 .

Although there was some association between the presence of limb motor impairment on admission and a worse outcome, the presence of limb motor deficits was not an independent predictor of aphasia recovery. Rather, aphasia and motor signs both reflect stroke severity.

We acknowledge limitations to the present study namely its retrospective design, the missing imaging data in some cases, the lack of compulsory MRI imaging that would give a better estimate of lesion size and number, the small subset of patients with standardized aphasia testing, and the lack of long-term follow up. Its strengths are the large series of patients included, the analysis of lesion size requiring only low technology as well as the use of a more comprehensive assessment tool.

The observation period of this study is long. However, the only change in patient management was the extension of the therapeutic window for thrombolysis from 3 to $4.5 \mathrm{~h}$.

Although most patients with aphasia are expected to improve beyond the first week of stroke, the ability to predict aphasia recovery in the acute stage is valuable for patients, families, and clinicians. Families often seek reassurance about patient's recovery during their first encounter with the attending physician, who will have to estimate it from a limited number of measures. On the other hand, physicians need to anticipate 
the length of admission and rehabilitation needs. Hence, longitudinal studies are necessary to provide more definite answers about the outcome and to understand the role of endovascular therapy in acute aphasia recovery.

In conclusion, there is a tendency for an early recovery of aphasia during the first week after thrombolysis for acute stroke, which will be complete in about $30 \%$ of patients. A composite verbal score - which includes the NIHSS_best Language, Commands and Questions items - seems to capture better the overall linguistic performance of patients with aphasia than NIHSS_Language item alone. Lesion size, initial aphasia severity, and vessel patency are the main predictors of improvement.

\section{Acknowledgment}

The authors thank Dr Bruno Miranda for his careful review of the English language.

\section{Funding}

No funding reported.

\section{Conflict of interest}

The authors report no conflict of interest.

\section{References}

1. Wade DT, Hewer RL, David RM, Enderby PM. Aphasia after stroke: natural history and associated deficits. J Neurol Neurosurg Psychiatry 1986;49:11-6.

2. Kauhanen ML, Korpelainen JT, Hiltunen P et al. Aphasia, depression, and non-verbal cognitive impairment in ischaemic stroke. Cerebrovasc Dis 2000;10:455-61.

3. Pedersen PM, Jørgensen HS, Nakayama H, Raaschou HO, Olsen TS. Aphasia in acute stroke: incidence, determinants, and recovery. Ann Neurol 1995;38:659-66.

4. Engelter ST, Gostynski M, Papa S et al. Epidemiology of aphasia attributable to first ischemic stroke incidence, severity, fluency, etiology, and thrombolysis. Stroke 2006;37:1379-84

5. Inatomi Y, Yonehara T, Omiya S, Hashimoto Y, HirAno T, Uchino M. Aphasia during the acute phase in ischemic stroke. Cerebrovasc Dis 2007;25:316-23.

6. Cropuelois A, Bogousslavsky J. Stroke aphasia: 1,500 consecutive cases. Cerebrovasc Dis 2010;31:392-9.

7. Röther J, Schellinger PD, Gass A et al. Effect of intravenous thrombolysis on MRI parameters and functional outcome in acute stroke $<6$ hours. Stroke 2002;33:2438-45.

8. Labeyrie MA, Turc G, Hess A et al. Diffusion Lesion Reversal After Thrombolysis A MR Correlate of Early Neurological Improvement. Stroke 2012;43:2986-91.

9. Seitz RJ, Sondermann V, Wittsack HJ, Siebler M. Lesion patterns in successful and failed thrombolysis in middle cerebral artery stroke. Neuroradiology 2009;51:865-71.
10. Lazar RM, Speizer Ae, Festa JR, Krakauer JW, MarSHALL RS. Variability in language recovery after first-time stroke. J Neurol Neurosurg Psychiatry 2008;79:530-4.

11. Kremer C, Perren F, Kappelin J, Selariu E, AbulKasim K. Prognosis of aphasia in stroke patients early after iv thrombolysis. Clin Neurol Neurosurg 2013;115: 289-92.

12. Denier C, Flamand-Roze C, Dib F et al. Aphasia in stroke patients: early outcome following thrombolysis. Aphasiology 2015;29:442-56.

13. Lyden P, LU M, JACKSON $\mathrm{C}$ et al. Underlying structure of the National Institutes of Health Stroke Scale results of a factor analysis. Stroke 1999;30:2347-54.

14. Denier C, Chassin O, Vandendries $\mathrm{C}$ et al. Thrombolysis in stroke patients with isolated aphasia. Cerebrovas Dis 2016;41:163-9.

15. Pexman JW, Barber PA, Hill MD et al. Use of the Alberta Stroke Program Early CT Score (ASPECTS) for assessing CT scans in patients with acute stroke. AJNR Am J Neuroradiol 2001;22:1534-42.

16. Ferro JM. Neurologia do comportamento. Estudo de correlação com a tomografia axial computorizada. Doctoral thesis. Lisbon Faculty of Medicine. 1986.

17. Goodglass H, Kaplan E. The assessment of aphasia and related disorders. Philadelphia: Lea \& Febiger, 1972.

18. Ferro JM, Kertesz A. Comparative classification of aphasic disorders. J Clin Exp Neuropsychol 1987;9: 365-75.

19. Kertesz A. Western aphasia battery. San Antonio: The Psychological Corporation, 1982.

20. Saur D, Lange R, Baumgaertner A et al. Dynamics of language reorganization after stroke. Brain 2006;129: 1371-84.

21. Forkel SJ, de Schotten MT, Dell'Acqua $F$ et al. Anatomical predictors of aphasia recovery: a tractography study of bilateral perisylvian language networks. Brain 2014;137:2027-39.

22. Pulvermüller F, Hauk O, Zohsel K, Neininger B, Mohr B. Therapy-related reorganization of language in both hemispheres of patients with chronic aphasia. NeuroImage 2005;28:481-9.

23. Naeser MA, Martin PI, Nicholas M et al. Improved picture naming in chronic aphasia after TMS to part of right Broca's area: an open-protocol study. Brain Lang 2005;93:95-105.

24. Olivot JM, Mlynash M, Thiss VN et al. Relationships between infarct growth, clinical outcome, and early recanalization in diffusion and perfusion imaging for understanding stroke evolution (DEFUSE). Stroke 2008;39:2257-63.

25. Mikulik R, Dusek L, Hill MD et al. Pattern of response of National Institutes of Health Stroke Scale components to early recanalization in the CLOTBUST trial. Stroke 2010;41:466-70.

26. Hillis AE, Kane A, Tuffiash E et al. Reperfusion of specific brain regions by raising blood pressure restores selective language functions in subacute stroke. Brain Lang 2001;79:495-510.

27. Jacquin A, Virat-Brassaud ME, Rouaud $\mathrm{O}$ et al. Vascular Aphasia Outcome after Intravenous Recombinant Tissue Plasminogen Activator Thrombolysis for Ischemic Stroke. Eur Neurol 2014;71:288-95.

28. Cappa SF, Perani D, Grassi $F$ et al. A PET follow-up study of recovery after stroke in acute aphasics. Brain Lang 1997;56:55-67. 